\title{
Impact of maternal antepartum depressive and anxiety symptoms on birth outcomes and mode of delivery: a prospective cohort study in east and west coasts of Malaysia
}

Hashima E. Nasreen ${ }^{1 *}$ D, Hafizah Binti Pasi ${ }^{1+}$, Sakinah Md Rifin ${ }^{2 \dagger}$, Mohd Aznan Md Aris ${ }^{2+}$, Jamalludin Ab Rahman ${ }^{1+}$, Razman Mohd Rus ${ }^{1+}$ and Maigun Edhborg ${ }^{3+}$

\begin{abstract}
Background: Antepartum depressive and anxiety symptoms (ADS and AAS) are prevalent in Malaysia. Prior evidence linking maternal ADS and AAS with adverse birth outcomes and caesarean section (CS) or instrumental delivery is conflicting. There is no research in Malaysia on the association between maternal mental disorders and adverse birth outcomes and mode of delivery. This study aims to investigate the independent effect of maternal ADS and AAS on low birth weight (LBW), preterm birth (PTB) and CS or instrumental delivery among women in east and west coasts of Malaysia.

Methods: We used data from a prospective cohort study of 799 pregnant women from health clinics of two states in east and west coasts of Malaysia. Baseline data were measured at the third trimester of pregnancy on ADS, AAS, socioeconomic condition, anthropometric status, reproductive history and intimate partner violence. Birth outcomes and mode of delivery were determined at the time of delivery. Univariate and multiple Cox's regressions were applied to assess the association between ADS and AAS and LBW, PTB and CS or instrumental delivery.

Results: ADS was significantly associated with an increased risk of giving birth to LBW babies in both east coast $(\mathrm{RR}=3.64 ; 95 \% \mathrm{Cl} 1.79-7.40)$ and west coast $(\mathrm{RR}=3.82 ; 95 \% \mathrm{Cl} 1.86-7.84)$, but not with PTB. AAS was associated with increased risk of both $\mathrm{LBW}(\mathrm{RR}=2.47 ; 95 \% \mathrm{Cl} 1.39-4.38)$ and PTB $(\mathrm{RR}=2.49 ; 95 \% \mathrm{Cl} 1.16-5.36)$ in the east coast, but not in west coast. The risk of CS or instrumental delivery was evident among women with ADS (RR $=2.44 ; 95 \%$ Cl 1.48-4.03) in west coast only.

Conclusion: ADS predicts LBW in both coasts, AAS predicts LBW and PTB in east coast, and ADS predicts CS or instrumental delivery in west coast. Policies aimed at detection and management of ADS and AAS during antenatal check-up in health clinics may help improve birth outcomes and reduce obstetric interventions.
\end{abstract}

Keywords: Antepartum depressive symptoms, Antepartum anxiety symptoms, Low birth weight, Preterm birth, Caesarean section, Instrumental delivery, Malaysia

\footnotetext{
* Correspondence: drnasreen@iium.edu.my

${ }^{\dagger}$ Hafizah Binti Pasi, Sakinah Md Rifin, Mohd Aznan Md Aris, Jamalludin Ab

Rahman, Razman Mohd Rus and Maigun Edhborg contributed equally to this

work.

${ }^{1}$ Department of Community Medicine, Faculty of Medicine, International

Islamic University Malaysia, Jalan Sultan Ahmad Shah, 25200 Kuantan,

Pahang, Malaysia

Full list of author information is available at the end of the article
}

(c) The Author(s). 2019 Open Access This article is distributed under the terms of the Creative Commons Attribution 4.0 International License (http://creativecommons.org/licenses/by/4.0/), which permits unrestricted use, distribution, and reproduction in any medium, provided you give appropriate credit to the original author(s) and the source, provide a link to the Creative Commons license, and indicate if changes were made. The Creative Commons Public Domain Dedication waiver (http://creativecommons.org/publicdomain/zero/1.0/) applies to the data made available in this article, unless otherwise stated. 


\section{Background}

Depression and anxiety are common complications during pregnancy and childbearing age [1], and are recognized as factors that may adversely impact on maternal and neonatal outcomes [2]. The prevalence of antepartum depressive symptoms (ADS) is estimated at $15.6 \%$ in low and lower middle income countries [3], which is higher than that reported from high income countries (12\%) [4]. The pooled prevalence is $15.2 \%$ for any antepartum anxiety disorder and $22.9 \%$ for antepartum anxiety symptoms (AAS) [5]. Experiencing ADS or AAS may expose both mothers and infants to psychological risks, such as impaired bonding to the foetus [6] and physiological consequences including low intra-uterine growth, low birth weight (LBW; less than $2500 \mathrm{~g}$ ) and preterm birth (PTB; birth before 37 weeks of gestation) [7-10]. ADS or AAS may also affect the mode of delivery [11] and women's preference for a caesarean section (CS) [12]. LBW and PTB are the leading causes of neonatal and infant morbidity, mortality [13], and neurodevelopmental impairments and disabilities [14]. Globally, it is estimated that nearly 22 million newborns, accounting for $16 \%$ of all births, were born with LBW. The incidence of PTB has been estimated to be $9.6 \%$ of all births, which corresponds to 12.9 million PTB worldwide [15]. Approximately $85 \%$ of these burdens concentrate in Africa and Asia [16]. There are a number of well-known risk factors for LBW, PTB and CS delivery including preeclampsia, hypertension, gestational diabetes, and intimate partner violence (IPV) $[7,17,18]$.

Several systematic reviews and meta-analyses have been carried out showing inconsistency and inconclusive association among ADS, PTB and LBW [7-10]. From a metaanalyses of 29 studies Grote et al. [7] reported significant associations among ADS, LBW and PTB. Accortt et al. [9] included 95 studies between 1977 and 2013 in an updated systematic review and concluded that ADS was rarely associated with PTB in larger, well-controlled studies. Another systematic review and meta-analysis about ADS and adverse birth outcomes found PTB significantly associated with ADS, but not with LBW [8].

Since researchers focused more attention on ADS, less studies were done on AAS and birth outcomes. But the high level of comorbidity between ADS and AAS, estimated at $60 \%$ [4], suggests anxiety and depression should be examined concurrently. One systematic review explored the effect of antenatal psychological distress on PTB in 39 studies and included 14 studies examining the effect of ADS, four studies examining AAS, and five studies stress [1]. The findings suggest an increased risk of PTB when a woman experiences one or more of the described psychological disorders. However, Grigoriadis et al. [19] found in a recent systematic review and meta-analysis including 29 articles that AAS was associated with both PTB and LBW.

Data on the effect of depression and anxiety on mode of delivery are more limited [12]. Moreover, making an evidence-based decision has been challenging, particularly because of mixed and contradictory findings [20, 21]. In a systematic review of four studies exploring CS following exposure to AAS, three studies showed non-significant results and one significant [19]. However, Bayrampour et al. [11] found that ADS in the third trimester of pregnancy increased the risk of emergency CS, but not AAS.

Given the inconsistent results vis-à-vis the impact of ADS and AAS on adverse birth outcomes and mode of delivery across countries, and particularly less research on AAS, more evidence should be warranted to explore these associations in different settings to inform contextualized interventions. In Malaysia, there are no studies assessing the association among ADS, AAS, LBW, PTB and mode of delivery, where the incidences of LBW and PTB were 11.0 and $11.3 \%$, respectively in 2012 [15], and increased trend of CS delivery from $10.5 \%$ in 2000 to $15.7 \%$ in 2006 [22]. This prospective cohort study addressed this shortcoming by examining the impact of ADS and AAS on LBW, PTB and CS or instrumental delivery among women in east and west coasts of Malaysia.

\section{Methods}

\section{Study design and setting}

Data for this prospective cohort study were collected at the third trimester of pregnancy and at birth from a larger longitudinal study of perinatal depressive and anxiety symptoms among women in Malaysia. The women were recruited from health clinics in Pahang and Selangor states in the east and west coasts of peninsular Malaysia, respectively [23]. Pahang is predominated by the indigenous and rural culture, which has a population about 1.5 million. The economy in Pahang is primarily based on agriculture and mining, contributing $4.2 \%$ to country's gross domestic product (GDP). The majority of women are involved in unpaid domestic work. Conversely, Selangor is more developed, predominantly urban and contemporary. It has a population of about 5.5 million and is the leading GDP contributor (22.6\%) to national economy, where the major sources of economy are commerce, industry and service sectors [24]. Pregnant women in Malaysia get free antenatal and postnatal care at the government health clinics and hospitals, and majority of the births occur at hospitals [23].

\section{Participants and sample size}

Participants were pregnant women enrolled in this study at their third trimester of pregnancy ( $\geq 28$ weeks) from 10 health clinics where the highest number of attendance for 
antenatal check-up was observed. Estimation of gestational weeks at enrolment was based on ultrasound scanning during antepartum check-up. A cohort of 905 pregnant women were recruited for the study, who were followed-up until delivery, and delivery information was collected from 799 (88.3\%) singleton live births (Fig. 1). The exclusion criteria for the study were non-Malaysian, illiterate, moved out from the study area, multiple birth (as it affects birth outcomes) and intrauterine death. The details about sample size for the study were explained elsewhere [23].

\section{Data collection}

Data for the larger longitudinal study were collected from March 2016 to August 2017. Baseline data were collected at third trimester of pregnancy on socioeconomic and anthropometric status, reproductive history, perceived social support, IPV, depression in earlier pregnancy, and depressive and anxiety symptoms. We collected baseline data through self-reported structured questionnaires in Malay during women's antenatal care visits at the health clinics. Obstetric data were collected on newborn's sex, gestational age at delivery, mode of delivery, complications during delivery, live or still birth, birth weight and height from the child health cards of respective health clinics. In Malaysia, all obstetric information including anthropometric measurements of newborn babies are measured within $2-48 \mathrm{~h}$ after delivery by the attending health personnel of hospitals where the deliveries took place. All these data and their subsequent growth charts are then recorded in the child health cards of the respective health clinics. Trained nurses distributed the baseline questionnaires to the participating women and gave them information about the study if needed. The nurses and research assistants were trained on the questionnaire and data collection, and they scrutinized the completed questionnaires on the spot for any missing data. The baseline questionnaires were pretested among pregnant women during their antenatal care visits at the International Islamic University Malaysia's family health clinic and finalized based on feedback received in the field test.

\section{Measures}

The outcome variables used in this study include LBW, PTB and SC or instrumental delivery. LBW was classified according to the World Health Organization definition as newborn babies with a birth weight of $<2500 \mathrm{~g}$ and PTB was defined as babies born before 37 weeks of gestation [13]. Mode of delivery was categorized as spontaneous vaginal and CS or instrumental delivery encompassing forceps delivery or vacuum extraction.

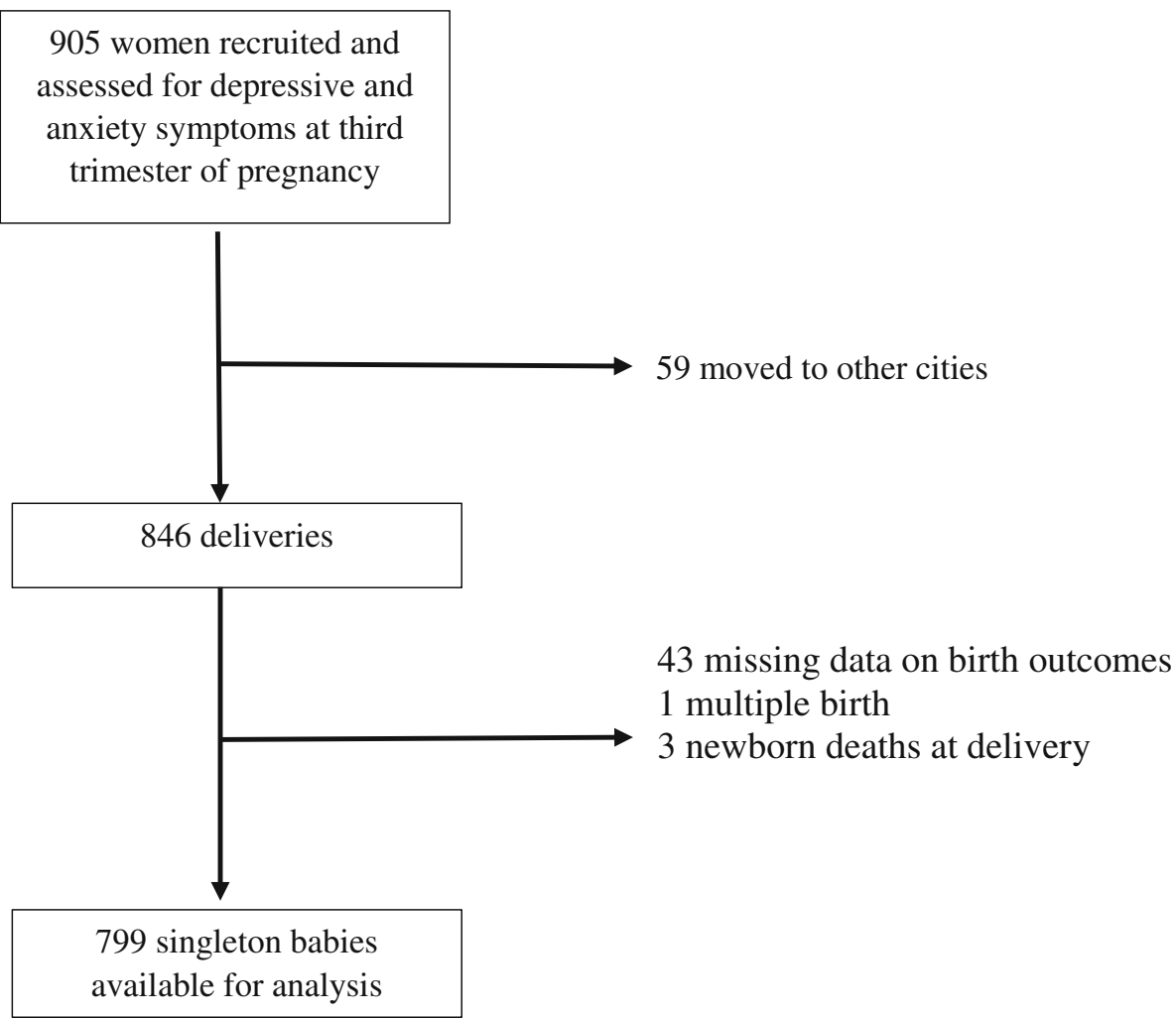

Fig. 1 Study participants 
The Edinburgh Postnatal Depression Scale (EPDS) was used to assess maternal depressive symptom [25]. The EPDS is a 10 items questionnaire, rated $0-3$ on each item and ranging from 0 to 30 . The scale rates the intensity of depressive symptoms during the previous 7 days, a higher score indicates more depressive symptoms. The items assessed dysphoric mood (five items), anxiety (two items), guilt (one item), ability to cope with everyday life (one item), and suicidal thought (one item). The EPDS is validated in Malay, reporting sensitivity to be $72.7 \%$, specificity $95 \%$, and positive predictive value $80 \%$, using 11.5 as the cut-off score [26]. Thus, the cut-off score $\geq 12$ was used to categorise pregnant women with depressive symptoms in this study. The Cronbach's alpha of the scale was 0.75 at third trimester of pregnancy.

The anxiety sub-scale of validated Malay version of Depression, Anxiety and Stress Scale (DASS 21) was used to measure maternal anxiety symptoms over the previous week [27]. DASS 21 comprises of 21 questions, subdivided into three domains: depression, anxiety and stress, with seven questions in each domain. The choice of response varied from 0 ('did not apply at all') to 3 ('applied very much' or 'most of the time'). In this research we used only the seven anxiety items. As we used the short version of DASS with 21 items, instead of 42, the score on the DASS 21 anxiety scale was multiplied by 2 to calculate final score of anxiety symptoms. The cut-off point $\geq 8$ was used in the study to estimate the prevalence of AAS [28]. The DASS 21 anxiety scale showed a relatively good reliability with Cronbach's alpha of 0.74 .

Socio-demographic data include women's age, education (primary, secondary or tertiary), occupation (homemaker or employed encompassing government employee, non-government employee and self-employed), and monthly household income ( $>$ RM 5599 as high income, RM 2300-5599 as middle income or $<\mathrm{RM}$ 2300 as low income) [24]. Parity (primi or multipara) and newborn baby's sex (girl or boy) were also recorded. Body mass index (BMI) was defined as women's weight in kilogram divided by the square of height in meter and was categorized as underweight $\left(<18.5 \mathrm{~kg} / \mathrm{m}^{2}\right)$, normal $\left(18.5-24.9 \mathrm{~kg} / \mathrm{m}^{2}\right)$ or overweight/obese $\left(\geq 25.0 \mathrm{~kg} / \mathrm{m}^{2}\right)$ [29]. The validated Malay version of Multidimensional Scale of Perceived Social Support (MSPSS) was used to measure social support [30]. MSPSS consists of 12 items, scored on a 7-point scale from 1 (very strongly disagree) to 7 (very strongly agree), with a total score ranging from 1 to 84 , a higher score indicates more support. MSPSS showed good internal consistency in this study with the Cronbach's alpha of 0.93 .

IPV was defined by a lifetime experience of physical abuse ever and physical abuse during pregnancy by husbands. Physical abuse includes four items, scored yes (1) and no (0): 1) slapped or thrown object at her, 2) pushed or shoved to the ground, 3) punched or hit, 4) kicked or dragged on the ground [31]. The total score of physical abuse ranged from 0 to 4 and categorized as no act of physical abuse (0) and acts of physical abuse (1-4).

\section{Statistical analysis}

We compared the respondents' baseline characteristics by sites using independent sample $t$ test, chi-square or Fisher's exact test. Prevalence of ADS and AAS, and incidence of LBW, PTB and CS or instrumental delivery were calculated. Univariate Cox's regression analyses were carried out to identify possible risk factors of LBW, PTB and CS or instrumental delivery with $p<0.05$. To control the confounding effects of these possible risk factors on the association between ADS and AAS, and LBW, PTB and CS or instrumental delivery, adjusted risk ratios (aRR) were computed using multiple Cox's regression analyses. Statistical significance of the RR was tested by confidence interval (CI) at $95 \%$. Because of the main exposure variables, ADS and AAS were entered in all models regardless of univariate statistical significance level. Separate multiple Cox's regression analyses were performed to show the role of ADS (model I) and AAS (model II) as risk factors of LBW, PTB and CS or instrumental delivery. To examine the moderating effects of site (east coast vs. west coast), stratified analyses were conducted. Any violation of assumptions was observed by examining the collinearity between explanatory variables and outliers in the models.

\section{Results}

The final sample included 799 pregnant women $(55.8 \%$ form east coast and $44.2 \%$ from west coast) with mean age of $29.7( \pm 4.7)$ years in the third trimester of pregnancy. Women in west coast were more educated and employed than women in east coast. Approximately 51\% of the participants were from middle income level with median monthly household income of RM 3500 and $21 \%$ from high income level with significantly higher proportion fitted in west coast. The average BMI of pregnant women was $27.4( \pm 5.7) \mathrm{kg} / \mathrm{m}^{2}$ and $36 \%$ were primiparas. Twenty-one (2.6\%) and $13(1.6 \%)$ pregnant women reported of being the victim of at least one act of physical abuse ever and physical abuse during current pregnancy, respectively. There were $12.3 \%$ women suffering depressive symptoms and $28.7 \%$ anxiety symptoms during pregnancy. The prevalence of AAS was found to be higher in west coast than in east coast ( $35.1 \%$ vs. $23.5 \%$, respectively) (Table 1 ).

The average birth weight of a newborn baby was $3.0( \pm$ $0.5) \mathrm{kg}$ and the gestational age at delivery was $39.1( \pm 1.5)$ weeks. The percentage of pregnant women having LBW and PTB were $12.6 \%(n=93)$ and $6.1 \%(n=49)$, 
Table 1 Baseline characteristics of women at third trimester of pregnancy (in percent unless otherwise stated)

\begin{tabular}{|c|c|c|c|c|}
\hline & \multirow{2}{*}{$\begin{array}{l}\text { East coast } \\
N=446\end{array}$} & \multicolumn{2}{|c|}{ West coast } & \multirow[t]{2}{*}{$p$ value } \\
\hline & & $\overline{\mathrm{N}^{a}}$ & $N=353$ & \\
\hline Age, mean (SD) & $29.8(5.1)$ & 350 & $29.6(4.1)$ & 0.525 \\
\hline Education & & 352 & & \\
\hline Primary & 4.0 & & 0.6 & $<0.001$ \\
\hline Secondary & 51.1 & & 32.1 & \\
\hline Tertiary & 44.8 & & 67.3 & \\
\hline Occupation & & 353 & & \\
\hline Homemaker & 49.1 & & 35.7 & $<0.001$ \\
\hline Employed & 50.9 & & 64.3 & \\
\hline Monthly household income & & 353 & & \\
\hline Low income & 36.3 & & 17.8 & $<0.001$ \\
\hline Middle income & 48.0 & & 54.4 & \\
\hline High income & 15.7 & & 27.8 & \\
\hline BMI & & 353 & & \\
\hline Underweight & 6.3 & & 4.2 & 0.431 \\
\hline Normal weight & 29.8 & & 31.4 & \\
\hline Overweight/obesity & 63.9 & & 64.3 & \\
\hline Parity & & 353 & & \\
\hline Primipara & 31.4 & & 37.1 & 0.090 \\
\hline Multipara & 68.6 & & 62.9 & \\
\hline \multicolumn{5}{|l|}{ Intimate partner violence } \\
\hline Physical abuse ever & 2.5 & 351 & 2.8 & 0.738 \\
\hline $\begin{array}{l}\text { Physical abuse during } \\
\text { pregnancy }\end{array}$ & 1.6 & 352 & 1.7 & 0.881 \\
\hline Social support, mean (SD) & $\begin{array}{l}65.6 \\
(10.3)\end{array}$ & 353 & $\begin{array}{l}67.8 \\
(10.2)\end{array}$ & 0.003 \\
\hline Depression in earlier pregnancy & 6.7 & 350 & 14.0 & 0.001 \\
\hline Antepartum depressive symptoms & 11.2 & 353 & 13.6 & 0.307 \\
\hline Antepartum anxiety symptoms & 23.5 & 353 & 35.1 & $<0.001$ \\
\hline
\end{tabular}

${ }^{\mathrm{a} D a t a}$ available for analysis

respectively. A total of $26.5 \%(n=212)$ of our sample had either CS or instrumental delivery. However, no significant differences were noted between sites in the incidence of LBW $(11.7 \%$ in east coast vs. $11.6 \%$ in west coast, $p=0.984)$, PTB $(6.5 \%$ in east coast vs. $5.7 \%$ in west coast, $p=0.625)$ and CS or instrumental delivery $(26.5 \%$ in east coast vs. $26.6 \%$ in west coast, $p=0.957$ ). Of the 26 (3.3\%) women experienced complications during delivery, $84.6 \%$ had CS or instrumental delivery. Complications during delivery was found to be higher in women with ADS (8.2\%, $p=0.003)$ and AAS $(5.7 \%, p=0.014)$ than women without ADS (2.6\%) and AAS (2.3\%). Depressed women experienced foetal distress and prolonged second stage of labour, while anxious women experienced foetal distress, prolonged second stage of labour, oligohydramnios or type 2 diabetes (not shown).
Univariate regression analyses show that ADS is positively associated with LBW, PTB and CS or instrumental delivery, and AAS with LBW and PTB but not with CS or instrumental delivery. Participants from low income households, who were underweight, who experienced physical abuse ever and/or during pregnancy reported increased risk of LBW and PTB. Primipara women were more likely to give birth to LBW babies and to have CS or instrumental delivery. Women who had social support reported a decreased risk of PTB. Meanwhile, higher BMI of pregnant women, complications during delivery and gestational age were significant predictors for CS or instrumental delivery as compared to others (Table 2).

Tables 3, 4 and 5 show the associations between ADS and AAS and LBW, PTB and CS or instrumental delivery. After adjusted with all factors that were significant in the crude analysis, pregnant women with ADS had an increased risk of giving birth to babies with LBW (RR 3.58; 95\% CI 2.16-5.94) as compared to women without depressive symptoms in the final model, but women with AAS did not. Further stratified analyses show that the association between ADS and LBW remained with the modest changes in risks in both east coast (RR 3.64; 95\% CI 1.79-7.40) and west coast (RR 3.82; 95\% CI 1.86-7.84). However, AAS had emerged as a risk factor for LBW in east coast (RR 2.47; 95\% CI 1.39-4.38) and no association between ADS and LBW existed in west coast. Women's older age, low and middle income, primipara, women's underweight and physical abuse ever were also associated with LBW in west coast, and only women's underweight in east coast (Table 3).

In the final model, ADS was independently associated with PTB (RR 2.36; 95\% CI 1.12-4.99), but AAS was not (Table 4). Conversely, after stratification, ADS was no longer associated with PTB, neither in east coast nor in west coast. However, AAS was found to be an independent risk factor for PTB in east coast (RR 2.49; 95\% CI 1.16-5.36) together with women's underweight. Physical abuse ever was the only risk factor for PTB in west coast (Table 4).

With respect to the mode of delivery, the final model reveals that women with ADS had 55\% increased risk for giving birth through CS or instruments. In the stratified analyses, a stronger RR was found in the association between ADS and giving birth through CS or instruments in west coast, but not in east coast. AAS was not associated with increased risk of giving birth through CS or instruments, neither in final model nor in stratified model. Complications during delivery was a strong risk factor for CS or instrumental delivery in both sites. The risk of CS or instrumental delivery increased with primipara and women's higher BMI, and decreased with higher gestational age in east coast (Table 5). 
Table 2 Univariate analyses between potential confounders and outcomes under study $(N=799)$

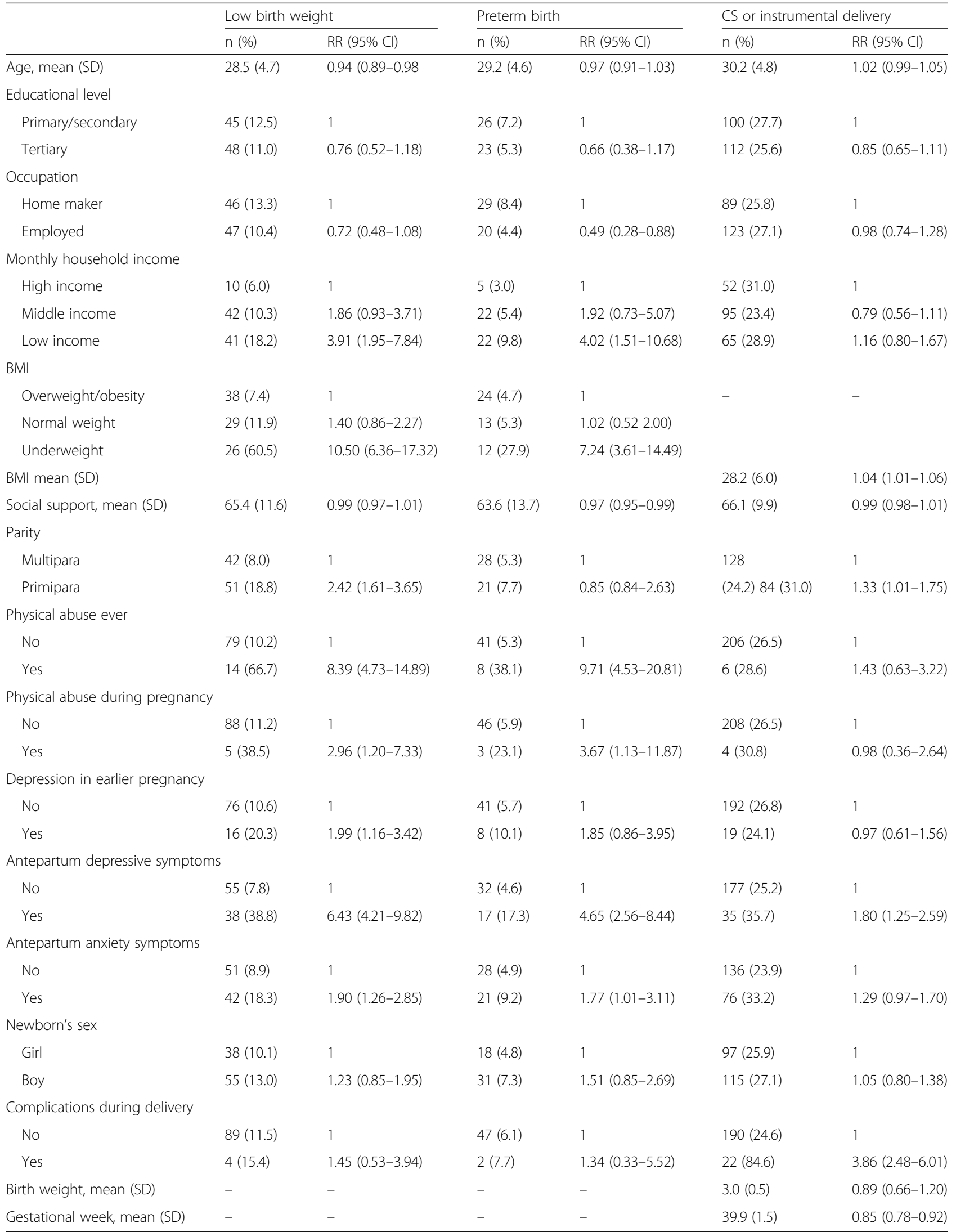


Table 3 Association between antepartum depressive and anxiety symptoms and LBW

\begin{tabular}{|c|c|c|c|}
\hline & \multirow{2}{*}{$\begin{array}{l}\text { Final model } \\
\text { aRR }(95 \% \mathrm{Cl})\end{array}$} & \multicolumn{2}{|l|}{ Stratified model } \\
\hline & & $\begin{array}{l}\text { East coast } \\
\text { aRR }(95 \% \mathrm{Cl})\end{array}$ & $\begin{array}{l}\text { West coast } \\
\text { aRR }(95 \% \mathrm{Cl})\end{array}$ \\
\hline \multicolumn{4}{|l|}{ Model I } \\
\hline Women's age & - & - & $1.12(1.01-1.24)$ \\
\hline Middle income & - & - & $5.07(1.65-15.59)$ \\
\hline Low income & $2.18(1.02-4.67)$ & - & $5.01(1.25-20.15)$ \\
\hline Primipara & $2.46(1.62-3.71)$ & $2.00(1.23-3.54)$ & $5.17(2.29-11.72)$ \\
\hline Underweight & $3.82(2.07-7.03)$ & $4.69(2.24-9.79)$ & $6.12(1.69-28.05)$ \\
\hline Physical abuse ever & $2.41(1.22-4.74)$ & - & $7.18(2.00-18.64)$ \\
\hline Antepartum depressive symptoms & $3.58(2.16-5.94)$ & $3.64(1.79-7.40)$ & $3.82(1.86-7.84)$ \\
\hline \multicolumn{4}{|l|}{ Model II } \\
\hline Women's age & - & - & $1.12(1.01-1.24)$ \\
\hline Middle income & - & - & $4.61(1.57-13.56)$ \\
\hline Low income & $2.48(1.18-5.20)$ & - & $5.82(1.61-21.09)$ \\
\hline Primipara & $2.35(1.55-3.56)$ & - & $5.75(2.59-12.77)$ \\
\hline Underweight & $5.48(3.03-9.78)$ & $7.92(4.17-15.04)$ & $7.84(2.71-22.66)$ \\
\hline Physical abuse ever & $4.52(2.36-8.66)$ & - & $14.76(5.63-38.67)$ \\
\hline Antepartum anxiety symptoms & - & $2.47(1.39-4.38)$ & - \\
\hline
\end{tabular}

\section{Discussion}

In this study we analysed the associations between depressive and anxiety symptoms in the third trimester of pregnancy and LBW, PTB and CS or instrumental delivery in a cohort of Malaysian women in east and west coasts. We found that of the newborns $12.6 \%$ were born with $\mathrm{LBW}$ and $6.1 \%$ were PTB. ADS was an independent risk factor for LBW in the final model as well as in both coasts. According to PTB, the significant association was observed in the final model, but not in stratified models, neither in the east nor in the west coast. This association is independent of the effect of maternal nutritional and socioeconomic status (SES). Our results are in agreement with a meta-analysis, where Grote et al. [7] found a strong association between ADS and LBW, and that the risk was higher in low and middle income than in high income countries. Accortt et al. [9] also confirmed our results in their systematic review and reported that more studies (53\%) showed significant associations between ADS and LBW than between ADS and PTB (23\%). They concluded that ADS appeared to be a greater risk factor for LBW than PTB. Studies from Bangladesh [32] and South Africa [18] reported similarly that ADS was more consistently predicted LBW, but not PTB. However, our results are contrasted by, for example, Grigoriadis et al. [8], Jarde et al. [10] and Staneva et al. [1], who found no significant association between ADS and LBW, instead they found ADS associated with PTB. Nevertheless, it is difficult to determine whether there is an etiological heterogeneity across these settings, because of the

Table 4 Association between antepartum depressive and anxiety symptoms and PTB

\begin{tabular}{|c|c|c|c|}
\hline & \multirow{2}{*}{$\begin{array}{l}\text { Final model } \\
\text { aRR }(95 \% \mathrm{Cl})\end{array}$} & \multicolumn{2}{|l|}{ Stratified model } \\
\hline & & $\begin{array}{l}\text { East coast } \\
\text { aRR }(95 \% \mathrm{Cl})\end{array}$ & $\begin{array}{l}\text { West coast } \\
\text { aRR }(95 \% \mathrm{CI})\end{array}$ \\
\hline \multicolumn{4}{|l|}{ Model I } \\
\hline Underweight & $3.61(1.60-8.14)$ & $9.53(4.31-21.05)$ & - \\
\hline Physical abuse ever & $3.50(1.41-8.67)$ & - & $6.63(1.92-22.91)$ \\
\hline Antepartum depressive symptoms & $2.36(1.12-4.99)$ & - & - \\
\hline \multicolumn{4}{|l|}{ Model II } \\
\hline Underweight & $4.97(2.33-10.63)$ & $7.39(3.25-16.78)$ & - \\
\hline Physical abuse ever & $5.24(2.25-12.21)$ & - & $6.63(1.92-22.91)$ \\
\hline Antepartum anxiety symptoms & - & $2.49(1.16-5.36)$ & - \\
\hline
\end{tabular}


Table 5 Association between antepartum depressive and anxiety symptoms and caesarean section or instrumental delivery

\begin{tabular}{|c|c|c|c|}
\hline & \multirow{2}{*}{$\begin{array}{l}\text { Final model } \\
\text { aRR }(95 \% \mathrm{Cl})\end{array}$} & \multicolumn{2}{|l|}{ Stratified model } \\
\hline & & $\begin{array}{l}\text { East coast } \\
\text { aRR }(95 \% \mathrm{Cl})\end{array}$ & $\begin{array}{l}\text { West coast } \\
\text { aRR }(95 \% \text { Cl) }\end{array}$ \\
\hline \multicolumn{4}{|l|}{ Model I } \\
\hline Primipara & $1.34(1.02-1.76)$ & $1.47(1.01-2.15)$ & - \\
\hline Women's BMI & $1.04(1.02-1.06)$ & $1.03(1.01-1.06)$ & - \\
\hline Gestational age & $0.86(0.79-0.93)$ & $0.79(0.71-0.87)$ & - \\
\hline Complications during delivery & $3.29(2.09-5.20)$ & $6.10(3.30-11.26)$ & $2.05(1.03-4.08)$ \\
\hline Antepartum depressive symptoms & $1.55(1.06-2.26)$ & - & $2.44(1.48-4.03)$ \\
\hline \multicolumn{4}{|l|}{ Model II } \\
\hline Primipara & $1.33(1.01-1.75)$ & $1.47(1.01-2.15)$ & - \\
\hline Women's BMl & $1.04(1.02-1.06)$ & $1.03(1.01-1.06)$ & - \\
\hline Gestational age & $0.86(0.79-0.93)$ & $0.79(0.71-0.87)$ & - \\
\hline Complications during delivery & $3.73(2.40-5.81)$ & $6.10(3.30-11.26)$ & $2.84(1.47-5.47)$ \\
\hline Antepartum anxiety symptoms & - & - & - \\
\hline
\end{tabular}

different cultures, healthcare systems, and maternal and child health profile.

According to AAS, we found no significant association with LBW and PTB in the final models. But moderator analyses reveal that AAS had increased risks for both LBW and PTB in the east coast, but not in the west coast. Although AAS is reported to be more common in women with lower SES than in women with higher SES [7], more women in the west coast (35.1\%) reported AAS compared to women in the east coast (23.5\%). Despite, it was women with AAS and underweight in the east coast with lower SES compared to most prosperous women in west coast, who were more likely to give birth to LBW babies and PTB. This is consistent with a systematic review, where Ding et al. [33] reported that AAS was associated with increased risk of PTB and LBW, particularly among Asian women with low SES. Women's lower SES i.e. less education associated with less employment and low income may deleteriously affects women's health behaviour and mental state. Our results are consistent with a systematic review and meta-analysis [19] arguing the strength of relationship between AAS and LBW and PTB, hence replicating the results from Asian countries. Consistent with other research [19], our findings suggest that AAS (28.7\%) is more prevalent than ADS (12.3\%), it appears that the consequences of this disorder may be more adverse as well. Yang et al. [34] reported that only ADS or only AAS was not associated with LBW and PTB among Chinese women, but if participants had LBW with PTB and ADS and AAS co-occur, there was an increased risk. Therefore, public health efforts should address depressive and anxiety symptoms during pregnancy together with equal importance, since the comorbidity increases the adverse birth and neonatal outcomes.
Maternal underweight is a well-established risk factor for LBW and PTB in low and lower middle-income countries [7, 35], as evident in our results from both east and west coasts. However, contrary to women in the east coast, physical IPV ever has emerged as a risk factor for LBW and PTB in the west coast. In the context of traditional gender role in Asian culture, women in Malaysia are primarily expected to care for children and manage household chores, irrespective of whether they are working outside home or not [36]. Women in west coast living in a contemporary culture are empowered, as evident by higher education, more women employed in service sectors and higher income, which may challenge the traditional views of gender role and social norms and elucidate the occurrence of IPV. Although research on the impact of IPV on birth outcomes has yielded mixed results, a sizable body of research have found IPV associated with LBW [37, 38]. IPV may impact negatively on maternal coping behaviour, such as smoking, alcohol and substance use, poor maternal nutrition, limited prenatal care, inadequate weight gain, and elevating stress, anxiety or depressive symptoms [39]. These elevated psychological stresses might exacerbate pre-existing conditions, such as hypertension, gestational diabetes, or it may lead to other pregnancy complications [40]. Stresses of experiencing IPV also alter the hypothalamic-pituitary-adrenal axis leading to changes in hormones that may affect the infants to be born with LBW and PTB [39], in the same way as depression and anxiety during pregnancy influence infants to born with LBW $[1,7]$.

ADS, but not AAS was independently associated with CS or instrumental delivery in the final model, but a significant association was found among women with ADS in west coast after stratification. The other risk 
factor of CS or instrumental delivery in the west coast was complications during delivery, which was also the strongest risk factor for CS and instrumental delivery in east coast, indicating that the obstetric interventions were mostly medically indicated. It is confirmed by Karalasingam et al. [22] who reported that CS was conducted in Malaysia due to obstetric indications, such as diabetes, hypertension and breech babies. The rate of CS is increasing globally, including in Malaysia (23\%), despite WHO sets the target that CS rate should not exceed $15 \%$ in any population [22]. Research has shown mixed results about the association among ADS, AAS and CS, and most studies reported no association between mode of delivery and antepartum mental health $[20,41]$. Our findings support the association between ADS at the third trimester of pregnancy and the risk for emergency CS [11], and ADS at 32 weeks of gestation and CS and instrumental delivery [42]. The mechanism by which ADS interferes with the mode of delivery may be due to negative influences of maternal mood on women's confidence for delivery, which could contribute to fear of childbirth and lower pain threshold leading to prolonged labour and thus to increase prevalence of CS [43]. Our findings that women with ADS in the west coast were more likely to have CS is difficult to explain. Nonetheless the women with ADS experienced delivery complications, mainly the prolonged second stage of labour, which is one of the major causes for CS in west coast. Further research to explore the mechanism of how ADS impacts on prolonged labour is suggested. Moreover, women in west coast live in the most developed and modern part of Malaysia, with a life more alike with the western style and the prevalence of CS is always been high in western countries [11], depending towards more women preferring CS and, therefore, delivering by CS without medical indication [44].

The strengths of our study are that it includes a large number of pregnant women who were followed prospectively that helped us collect precise and reliable data. Our use of locally validated measures of ADS and AAS has increased validity of exposures. Although we adjusted for the effect of many priori confounders including IPV, the study has some limitations including an absence of the use of antidepressant medication during pregnancy [9] and lack of information on a number of variables such as anemia, hypertension, gestational diabetes and smoking (although smoking was uncommon among the women of our study population) [45]. Comorbidity was not considered in the results, which may confound the data as depression and anxiety often co-occur [34]. In addition, we have used categorical instead of continuous measures of ADS [7] and self-reported depression instead of clinical diagnosis, which could have increased the risk of PTB and LBW [33].

\section{Conclusion}

In this study we found independent associations of ADS with newborn's LBW, PTB and CS or instrumental delivery over and above the well-established risk factors of maternal underweight, low income and physical abuse ever, but not AAS. Taking into account the study sites, the association of ADS with LBW remains the same in both coasts, AAS becomes the predictor for both LBW and PTB in the east coast, and ADS for CS or instrumental delivery in the west coast. The clinical implication of this study is to integrate a universal screening intervention into antenatal care services in health clinics, where all pregnant women should be screened for ADS and AAS by the clinic nurses using locally validated EPDS and DASS-anxiety. Further, referring women with severe depressive and anxiety symptoms to the nearest psychiatric care facilities for accuracy of diagnosis and treatment of ADS and AAS combined with supporting women with IPV may help improve birth outcomes and reduce obstetric interventions.

\section{Abbreviations}

AAS: Antepartum anxiety symptoms; ADS: Antepartum depressive symptoms; BMI: Body mass index; CS: Cesarean section; DASS: Depression, Anxiety and Stress Scale; EPDS: Edinburgh Postnatal Depressive Scale; GDP: Gross domestic product; IPV: Intimate partner violence; LBW: Low birth weight;

MSPSS: Multidimensional Scale of Perceived Social Support; PTB: Preterm birth; SES: Socioeconomic status

\section{Acknowledgments}

The authors thank all clinic nurses and staff involved in helping the data collection. The authors also express gratitude to the women who participated in the study for generously giving their time and energy and providing personal details to the interviews. We would like to thank the Director General of Health, Malaysia for his permission to publish this article.

\section{Authors' contributions}

HEN, HBP, MAMA, JAR, RMR and ME participated in the planning and conception of the research questions and the study design. HEN was the principal investigator of the study and primarily conceptualized the research. HBP, SMR, MAMA and RMR were responsible for retrieving the data, and HEN was responsible for analysing the data. HEN and ME participated in interpreting the data and drafted the article. All authors critically revised the manuscript for important intellectual content. All authors read and approved the manuscript.

\section{Funding}

The study was supported by grants from the Ministry of Higher Education, Malaysia under the Fundamental Research Grant Scheme (FRGS15-238-0479).

\section{Availability of data and materials}

The dataset generated and analysed during the current study are not publicly available due to confidentiality issues but are available from the corresponding author on reasonable request.

\section{Ethics approval and consent to participate}

The study was approved by Malaysia Medical Research and Ethics Committee (NMRR-15-1902-28,439) and the Research Ethics Committee of International Islamic University Malaysia (IREC 555). Women were informed in detail about the study and written informed consent was obtained. Pregnant women with depressive symptoms were advised to visit the psychiatric department of a nearby hospital to get professional support. 


\section{Competing interests}

The authors declare that they have no competing interests.

\section{Author details}

'Department of Community Medicine, Faculty of Medicine, International Islamic University Malaysia, Jalan Sultan Ahmad Shah, 25200 Kuantan, Pahang, Malaysia. ${ }^{2}$ Department of Family Medicine, Faculty of Medicine, International Islamic University Malaysia, Jalan Sultan Ahmad Shah, 25200 Kuantan, Pahang, Malaysia. ${ }^{3}$ Department of Neurobiology, Care Sciences and Society, Karolinska Institute, SE-141 83 Huddinge, Stockholm, Sweden.

\section{Received: 3 February 2019 Accepted: 31 May 2019}

Published online: 14 June 2019

\section{References}

1. Staneva A, Bogossian F, Pritchard M, Wittkowski A. The effects of maternal depression, anxiety, and perceived stress during pregnancy on preterm birth: a systematic review. Women Birth. 2015:28(3):179-93.

2. Field T, Diego M, Hermandez-Reif M, Figueiiredo B, Deeds O, Ascencio A, et al. Comorbid depression and anxiety effect on pregnancy and neonatal outcomes. Infant Behav Dev. 2010;33(19):23-37.

3. Fisher J, Cabral de Mello M, Patel V, Rahman A, Tran T, Holton S, et al. Prevalence and determinants of common perinatal mental disorders in women in low- and lower-middle-income countries: a systematic review. Bull World Health Organ. 2012;90:139-49.

4. Bennett HA, Einarson A, Taddio A, Koren G, Einarson TR. Prevalence of depression during pregnancy: systematic review. Obstet Gynecol. 2004;103:698-708.

5. Dennis CL, Falah-Hassani K, Shiri R. Prevalence of antenatal and postnatal anxiety: systematic review and meta-analysis. Br J Psychiatry. 2017:210:315-23.

6. Tronick E, Reck C. Infants of depressed mothers. Harv Rev Psychiatry. 2009;17:147-56.

7. Grote NK, Bridge JA, Gavin AR, Melvin JL, lyengar S, Katon WJ. A metaanalysis of depression during pregnancy and the risk of preterm birth, low birth weight, and intrauterine growth restriction. Arch Gen Psychiatry. 2010; 67(10):1012-24

8. Grigoriadis S, VonderPorten EH, Mamisashvilli MSW, Tomlinson G, Dennis C$L$, Koren $G$, et al. The impact of maternal depression during pregnancy on perinatal outcomes: a systematic review and meta-analysis. J Clin Psychiatry. 2013;74(4):e321-41.

9. Accortt EE, Cheadle ACD, Schetter CD. Prenatal depression and adverse birth outcomes: an updated systematic review. Matern Child Health J. 2015;19:1306-37.

10. Jarde A, Morais M, Kingston D, Giallo R, McQueen GM, Giglia L, et al Neonatal outcomes in women with untreated antenatal depression compared with women without depression: a systematic review and metaanalysis. JAMA Psychiatry. 2016;73(8):826-37.

11. Bayrampour H, Salmon C, Vinturache A, Tought S. Effect of depressive and anxiety symptoms during pregnancy on risk of obstetric interventions. J Obstet Gynaecol Res. 2015;41(7):1040-8.

12. Kuo SY, Chen SR, Tzeng YL. Depression and anxiety trajectories among women who undergo an elective caesarean section. PLoS One. 2014:9:1e86653.

13. March of Dimes, Save the Children, WHO. Born too soon: the global action report on preterm birth. Geneva: WHO; 2012.

14. Wilson-Costello D, Friedman H, Minich N, Fanaroff AA, Hack M. Improved survival rates with increased neurodevelopmental disability for extremely low birth weight infants in the 1990s. Pediatrics. 2005;115:997-1003.

15. UNICEF data. Low birthweight. In: UNICEF; 2014. http://data.unicef.org/topic/ nutrition/low-birthweight/. Accessed 8 Jan 2019.

16. Beck S, Wojdyla D, Say L, Betran AP, Merialdi M, Harris RJ, et al. The worldwide incidence of preterm birth: a systematic review of maternal mortality and morbidity. Bull World Health Organ. 2010;88:31-8.

17. Schei B, Lukasse M, Ryding EL, Campbell J, Karro H, Kristjansdottir H, et al. A history of abuse and operative delivery, results from a European multicounty cohort study. PLoS One. 2014;9(1):e87579.

18. Brittain K, Myer L, Koen N, Koopowitz S, Donald KA, Barnett W, et al. Risk factors for antenatal depression and associations with infant birth outcomes. Paediatr Perinat Epidemiol. 2015;29(6):505-14.
19. Grigoriadis S, Graves L, Peer M, Mamisahvilli MSW, Tomlinson G, Vigod SN, et al. Maternal anxiety during pregnancy and the association with adverse perinatal outcomes: systematic review and meta-analysis. J Clin Psychiatry. 2018;79(5):17r12011.

20. Wu J, Viguera A, Riley L, Cohen L, Ecker J. Mood disturbance in pregnancy and the mode of delivery. Am J Obstet Gynecol. 2002;187:864-7.

21. Pavlov M, Steiner N, Kessous R, Weintraub Y, Sheiner E. Obstetric and neonatal outcome in patients with anxiety disorders. J Matern Fetal Neonatal Med. 2014;27(13):1339-42.

22. Karalasingam SD, Jegasothy $R$, Jeganathan R, Zolkepali NA, Aiman S. Rising caesarean section rates in Malaysia. Poster of National Obstetric Registry (NOR), Ministry of Health Malaysia. n.d. www.acrm.org.my/nor/doc/poster/ Rising_Caesarean_section rates_Poster.pdf. Accessed 10 Jan 2019.

23. Nasreen HE, Rahman JA, Rus RM, Kartiwi M, Sutan R, Edhborg M. Prevalence and determinants of antepartum depressive and anxiety symptoms in expectant mothers and fathers: results from a perinatal psychiatric morbidity cohort study in east and west coasts of Malaysia. BMC Psychiatry. 2018;18:195.

24. Government of Malaysia. Tenth Malaysia plan 2011-2015. Putrajaya: the economic planning unit, prime minister's department; 2010.

25. Cox JL, Holden JM, Sagovsky R. Detection of postnatal depression: development of the 10-item Edinburgh postnatal depression scale. $\mathrm{Br}$ J Psychiatry. 1987;150:782-6.

26. Azidah AK, Nordin R, Ismail SB, Yaacob MJ, Mustapha WMRW. Validation of the Malay version of Edinburgh postnatal scale. Asia Pac J Fam Med. 2004:3:9-18.

27. Ramli M, Mohd AF, Zaini Z. Translation, validation and psychometric properties of Bahasa Malaysia version of the depression, anxiety and stress scale (DASS). Asian J Psychiatry. 2007;8:82-9.

28. Lovibond SH, Lovibond PF. Manual for the depression, anxiety and stress scales. 2nd ed. Sydney: Psychology Foundation; 1995.

29. World Health Organization. Obesity: preventing and managing the global epidemics. WHO technical report series 894. Geneva: WHO; 2000.

30. Ng CG, Nurasikin MS, Loh HS, Anne Yee HA, Zainal NZ. Factorial validation of the Malay version of multidimensional scale of perceived social support among a group psychiatric patients. Malays J Psychiatry. 2012;21(2):1-10.

31. Garcia-Moreno C, Jansen HAFM, Ellsberg M, Heise L, Watts CH. Prevalence of intimate partner violence: findings from the WHO multi-country study on women's health and domestic violence. Lancet. 2006;368:1260-9.

32. Nasreen HE, Kabir ZN, Forsell Y, Edhborg M. Low birth weight in offspring of women with depressive and anxiety symptoms during pregnancy: results from a population based study in Bangladesh. BMC Public Health. 2010;10:515.

33. Ding XX, Wu YL, Xu SJ, Zhu RP, Jia XM, Zhang SF, et al. Maternal anxiety during pregnancy and adverse outcomes: a systematic review and metaanalysis of prospective cohort studies. J Affect Disord. 2014;159:103-10.

34. Yang S, Yang R, Liang S, Wang J, Weaver NL, Hu K, et al. Symptoms of anxiety and depression during pregnancy and their association with low birth weight in Chinese women: a nested case-control study. Arch Womens Ment Health. 2017;20:283-90.

35. UNICEF and WHO. Low birthweight: country, regional and global estimates. New York: UNICEF; 2004

36. Ahmad NA, Silim UA, Rosman A, Majdah M, Chan YY, Kasim NM, et al. Postnatal depression and intimate partner violence: a nationwide clinicbased cross-sectional study in Malaysia. BMJ Open. 2018:8:e020649. https:// doi.org/10.1136/bmjopen-2017-020649.

37. Chen $\mathrm{P}-\mathrm{H}$, Rovi S, Vega ML, Barett $\mathrm{T}$, Pan K-Y, Johnson MS. Birth outcomes in relation to intimate partner violence. J Natl Med Assoc. 2017:109(4):238-45

38. Sigalla GN, Mushi D, Meyrowitsch DW, Manongi R, Rogathi JJ, Gammeltoft T, et al. Intimate partner violence during pregnancy and its association with preterm birth and low birth weight in Tanzania: a prospective study. PLoS One. 2017;12(2):e0172540.

39. Alhusen JL, Ray E, Sharp P, Bullock L. Intimate partner violence during pregnancy: maternal and neonatal outcomes. J Women's Health. 2015; 24(1):100-6.

40. Coker AL, Sanderson M, Dong B. Partner violence during pregnancy and risk of adverse pregnancy outcomes. Paediatr Perinat Epidemiol. 2004;18:260-9.

41. Reck C, Zimmer K, Dubber S, Zipser B, Schlehe B, Gawlik S. The influence of general anxiety and childbirth-specific anxiety on birth outcome. Arch Womens Ment Health. 2013;16:363-9. 
42. Chung TK, Lau TK, Yip AS, Chiu HF, Lee DT. Antepartum depressive symptomatology is associated with adverse obstetric and neonatal outcomes. Psychosom Med. 2001;63(5):830-4.

43. Ryding EL, Wijma B, Wijma K, Rydhstrom H. Fear of child-birth during pregnancy may increase the risk of emergency caesarean section. Acta Obstet Gynecol Scand. 1998;77:542-7.

44. Stjernholm YV, Petersson K, Eneroth E. Change indications for caesarean sections. Acta Obstret Gynecol Scand. 2010;89:49-53.

45. Cnattingius $\mathrm{S}$. The epidemiology of smoking during pregnancy: smoking prevalence, maternal characteristics, and pregnancy outcomes. Nicotine Tob Res. 2004;6(Suppl 2):125-40.

\section{Publisher's Note}

Springer Nature remains neutral with regard to jurisdictional claims in published maps and institutional affiliations.

Ready to submit your research? Choose BMC and benefit from:

- fast, convenient online submission

- thorough peer review by experienced researchers in your field

- rapid publication on acceptance

- support for research data, including large and complex data types

- gold Open Access which fosters wider collaboration and increased citations

- maximum visibility for your research: over $100 \mathrm{M}$ website views per year

At BMC, research is always in progress.

Learn more biomedcentral.com/submissions 